\title{
Biologia de Dichomeris famulata Meyrick, 1914 (Lepidoptera: Gelechiidae) em milho
}

\author{
Biology of Dichomeris famulata Meyrick, 1914 (Lepidoptera: Gelechiidae) in maize
}

\author{
Luiz Henrique da Silva Fagundes Marques $^{\mathrm{I}}$ Octavio Nakano $^{\mathrm{II}}$ Vanderly Janeiro $^{\mathrm{III}}$ \\ Eduardo Augusto Girardi ${ }^{\mathrm{IV}}$ Gustavo Gonzaga Buenov
}

\section{RESUMO}

\begin{abstract}
Dichomeris famulata Meyrick, 1914 (Lepidoptera: Gelechiidae) é uma nova praga da espiga de milho no Brasil. sendo seu estudo importante em áreas de produção de sementes porque os grãos atacados pelas lagartas não germinam. $O$ objetivo deste trabalho foi estudar a sua biologia em condições de laboratório $\left(25 \pm 2{ }^{\circ} \mathrm{C}\right.$, UR de $65 \pm 10 \%$ e fotofase de 14 horas $)$. O ciclo biológico (ovo-adulto) foi de 35,2 dias. O período de incubação foi de 4,1 dias. A duração média da fase larval foi de 21,1 dias, sendo observados cinco instares larvais. A fase pupal durou 8,4 dias e o peso de pupa de machos e fêmeas foi de 12,4 e 11,3mg, respectivamente. As fêmeas colocaram, em média, 118 ovos, apresentando um periodo de pré-oviposição de 10,7 dias e de oviposição de 14,0 dias. A longevidade média de machos e fêmeas foi de 37,02 e 44,16 dias, respectivamente, e a razão sexual de 0,48. As lagartas danificam os estilo-estigmas e os grãos em estado leitoso por meio de pequenos orifícios de entrada, prejudicando o endosperma e principalmente a região do embrião, inutilizando-os para sementes. Os resultados obtidos neste trabalho fornecem subsidios para o estabelecimento de estratégias de manejo do inseto, especialmente em áreas de produção de sementes.
\end{abstract}

Palavras-chave: milho, ciclo-biológico, qualidade da semente, semente.

\section{ABSTRACT}

The caterpillar Dichomeris famulata Meyrick, 1914 (Lepidoptera: Gelechiidae) is a new pest of corn ear in Brazil, and its study is important in seed fields. The aim of this was to study the biology of this pest under laboratory conditions $\left(25 \pm 2{ }^{\circ} \mathrm{C}, 65 \pm 10 \%\right.$ of $\mathrm{RH}$ and 14-hours of photophase). The biological cycle (egg-adult) was of 35.2 days. The incubation period was of 4.1 days. The average larval development time was of 21.1 days, and 5 instars were observed. The pupal period was of 8.4 days and the pupae weight was of 12.4 and $11.3 \mathrm{mg}$ for males and females, respectively. The females laid an average of 118 eggs with a pre-oviposition period of 10.7 days and an oviposition time of 14.0 days. The mean longevity of males and females was of 37.02 and 44.16 days, respectively, and sex ratio was of 0.48 . The caterpillars damage the silks and grains at the milk stage through little entry orifices, damaging the endosperm and, more importantly, the embryo, reducing the seed germination. These results can support the establishment of strategies to manage this new corn ear pest, especially in seed fields.

Key words: corn, biological cycle, quality of seed, seed.

\section{INTRODUÇÃO}

O Brasil é o terceiro produtor mundial de milho (Zea mays L.) com uma produtividade média de, aproximadamente, 3,9t ha ${ }^{-1}$ (FNP, 2009). Uma das premissas para obtenção de elevadas produtividades na cultura do milho é a utilização de sementes de qualidade (CÍCERO, 1987; MARTIN et al., 2007a). A produção de sementes é um processo trabalhoso e de elevado custo, sendo necessário que as sementes

'Departamento de Entomologia e Acarologia, Escola Superior de Agricultura "Luiz de Queiroz" (ESALQ), Universidade de São Paulo (USP), Av. Pádua Dias 11, CP 9, 13418-900, Piracicaba, SP, Brasil. E-mail: luhsfm@gmail.com. Autor para correspondência. "Departamento de Entomologia e Acarologia, ESALQ, USP, Piracicaba, SP, Brasil.

"IIDepartamento de Estatística, Universidade Estadual de Maringá (UEM), Maringá, PR, Brasil.

${ }^{\mathrm{IV}}$ Embrapa Mandioca \& Fruticultura, Cruz das Almas, BA, Brasil.

vDow AgroScience Industrial, Jardinópolis, SP, Brasil. 
disponibilizadas para os agricultores possuam um nível de qualidade preconizado pela legislação, ou seja, maior que $80 \%$ de germinação (MARTIN et al., 2007b).

Uma das principais causas da queda na qualidade dos grãos e da baixa produtividade é a ocorrência de ataque de lagartas causando injúrias às espigas. As pragas que causam prejuízos na fase reprodutiva do milho são a lagarta-da-espiga, Helicoverpa zea (Boddie, 1850) (Lepidoptera: Noctuidae) e a lagarta do cartucho-do-milho, Spodoptera frugiperda (J. E. Smith, 1797) (Lepidoptera: Noctuidae) que danificam os grãos e, por meio dos orifícios abertos na espiga, facilitam a entrada de microrganismos, o que resulta em grãos ardidos (LUIZ \& MAGRO, 2007). Além destas, uma nova praga da espiga do milho tem sido observada nos últimos anos (2005-2009) (MARQUES, 2009). Trata-se da espécie Dichomeris famulata Meyrick, 1914 (Lepidoptera: Gelechiidae), uma lagarta pequena, que vive entre os grãos de milho atacando o endosperma e principalmente a região do embrião e, como consequência, inutilizando os grãos como semente. Assim, a ocorrência desta praga pode representar um problema grave nos campos de produção de sementes de milho, pois os danos passam muitas vezes despercebidos e comprometem a germinação (MARQUES, 2009).

No Brasil, esta praga tem sido encontrada no norte de São Paulo e sul de Minas Gerais (MARQUES, 2009; MENEZES et al., 2009). Devido à falta de informações sobre a biologia de $\boldsymbol{D}$. famulata no Brasil, desenvolveu-se a presente pesquisa, que objetivou estudar a biologia da referida espécie em milho.

\section{MATERIAL E MÉTODOS}

Os estudos foram realizados no Departamento de Entomologia e Acarologia da ESALQ/ USP, durante o período de fevereiro de 2007 a fevereiro de 2009, sob condições controladas de temperatura $\left(25 \pm 2^{\circ} \mathrm{C}\right)$, umidade relativa $(65 \pm 10 \%)$ e fotofase $(14$ horas). Os insetos coletados foram identificados como Dichomeris famulata, Meyrick, 1914 pelo Dr. Vitor Osmar Becker (Universidade de Brasília, Instituto de Ciências Biológicas, Departamento de Zoologia).

Para iniciar a criação em laboratório de $\boldsymbol{D}$.

famulata, foram coletadas, aproximadamente, 200 lagartas de diferentes ínstares, em plantas de milho, cultivares IAC 8333 e Dow 2B655, da área experimental da ESALQ/USP. As lagartas foram colocadas em placas de Petri $(60$ X $12 \mathrm{~mm})$ e alimentadas com estilo-estigmas e grãos leitosos. As pupas foram individualizadas, separadas por sexo, conforme BUTT \& CANTU (1962), e colocadas no interior de placas de acrílico, contendo papel filtro umedecido diariamente com água destilada, para manter a umidade. Após a emergência, foram individualizados casais em gaiolas cilíndricas de armação de ferro, revestidas de tecido voile, $c 0 \mathrm{~m} 14 \mathrm{~cm}$ de diâmetro e $19 \mathrm{~cm}$ de altura, com um papelão na base contendo um orifício no centro, onde foi introduzido um tubo cilíndrico de polietileno de $2,54 \mathrm{~cm}$ de diâmetro e $10 \mathrm{~cm}$ de comprimento, fechado na base por uma rolha, com um orifício na metade de seu comprimento. No interior do tubo, colocou-se solução de mel a $10 \%$ e no orifício foi colocado um chumaço de algodão hidrófilo que fornecia, por capilaridade, $\mathrm{o}$ alimento às mariposas. $\mathrm{Na}$ abertura superior do tubo cilíndrico, foi acondicionada a ponta da espiga de milho, como substrato de oviposição. As espigas contendo os ovos foram retiradas diariamente, e o alimento trocado a cada dois dias. Para o estudo da biologia, realizado com 150 lagartas, foram avaliados os seguintes parâmetros biológicos: duração e viabilidade das fases de ovo ( $2^{\mathrm{a}}$ postura), de lagarta, de pré-pupa e de pupa; peso de pupas com 24h (machos e fêmeas). Para adultos (25 casais), observaram-se: longevidade de machos e fêmeas, fecundidade, duração do período de préoviposição e oviposição. Determinaram-se, também, o número e a duração dos ínstares, realizando-se a medição diária da largura da cápsula cefálica de 30 lagartas, por meio de um medidor WILD MMS 235, acoplado a um microscópio estereoscópico. Para a determinação do número de ínstares, utilizou-se a curva multimodal de frequência de medidas de cápsula cefálica, sendo as hipóteses formuladas e testadas no modelo linearizado da regra de Dyar, através do software MOBAE (Modelos Bioestatísticos para a Entomologia) (HADDAD et al., 1995). Os dados de peso de pupas foram submetidos ao teste de normalidade Kolmogorov-Smirnov, e as médias comparadas pelo teste paramétrico t de Student, a 5\% de probabilidade, sendo essa análise efetuada pelo Software R (THE R PROJECT FOR STATISTICAL COMPUTING, 2009). A sobrevivência e longevidade dos adultos foram analisadas pelo modelo de Weibull (SGRILLO, 1982; COLOSIMO \& GIOLO, 2006). Foi adotado o delineamento experimental inteiramente casualizado para todos os parâmetros biológicos observados.

\section{RESULTADOS E DISCUSSÃO}

Ovo. Em laboratório, as posturas de $\boldsymbol{D}$. famulata são realizadas isoladamente nos estiloestigmas e principalmente nas rugosidades da palha da espiga de milho. Os ovos são elípticos e de coloração amarela, adquirindo tonalidade alaranjada com o desenvolvimento (Figura 1A). O período de 


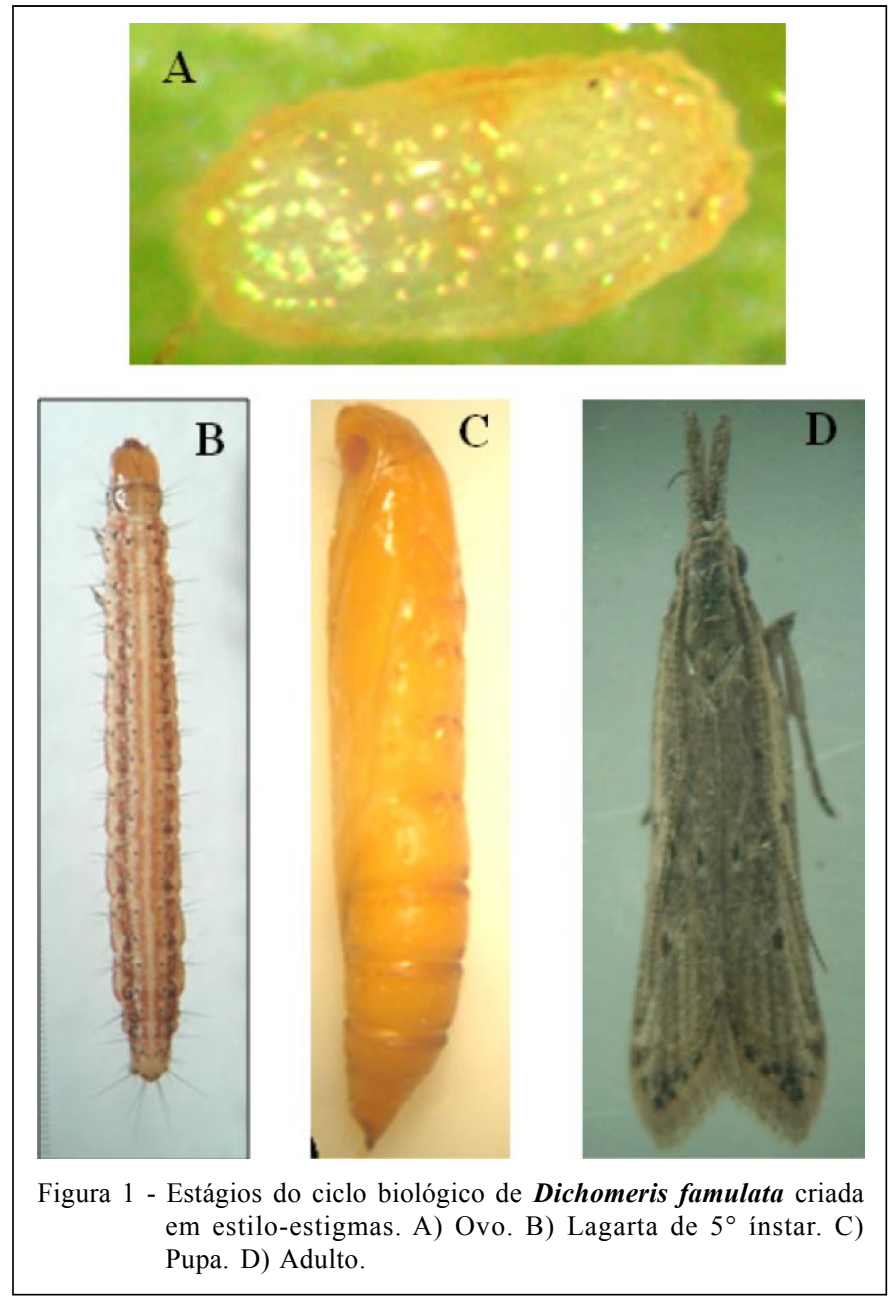

incubação foi de 4,1 dias, com viabilidade de 63,2\% (Tabela 1). Esse resultado está de acordo com CIRO et al. (1992), que estudaram a biologia da espécie com lagartas criadas em sorgo a $24^{\circ} \mathrm{C}$ e $74 \%$ de umidade relativa.

Lagarta. A lagarta (Figura 1B) é extremamente ágil e, quando tocada, pode se movimentar para frente ou para trás; não apresenta hábito canibal, podendose encontrar mais de uma lagarta por espiga. A duração e a viabilidade desta fase foram de 21,1 dias e $84 \%$ respectivamente, (Tabela 1 ), resultado semelhante ao obtido por CIRO et al. (1992), cuja fase larval média foi de 21,3 dias. A lagarta apresentou cinco ínstares (Figura 2 ), com durações de $4,1 \pm 0,31 ; 3,2 \pm 0,41 ; 2,8 \pm 0,68$;

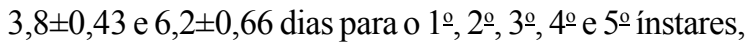
respectivamente, obedecendo à regra de Dyar (DYAR, 1890), ou seja, a largura das cápsulas cefálicas aumentou a cada ínstar, numa razão de 1,56 (a regra prevê uma variação de 1,1 a 1,9, com média de 1,4). Desta forma, os resultados apresentados coincidem com os dados obtidos por CIRO et al. (1992), que observaram a existência de cinco ínstares larvais de $\boldsymbol{D}$. famulata quando alimentadas com sorgo, em laboratório, a $24^{\circ} \mathrm{C}$. Portanto, aparentemente, o sorgo e o milho são hospedeiros nutricionalmente adequados para esta espécie-praga, pois não ocasionaram alterações no número de instares nos dois hospedeiros; a variação do número de ínstares pode ser uma indicação de adequação ou inadequação nutricional (PANIZZI; PARRA, 1991).

Pré-pupa. O início da fase de pré-pupa foi considerado a partir do momento em que as lagartas cessaram a alimentação, perderam a mobilidade e recobriram o corpo com um envoltório de seda, estiloestigmas e resíduos da alimentação. A duração da fase de pré-pupa foi, em média, de 1,6 dias e a viabilidade de 100\% (Tabela 1).

Pupa. A pupa inicialmente é amarelada, encerrada em um casulo formado de seda, contendo detritos de alimentação aderidos a ela (Figura 1C). Com o passar do tempo, a pupa vai adquirindo uma tonalidade mais escura. A fase de pupa apresentou uma 
Tabela 1 - Duração ( \pm EP) e viabilidade das fases de ovo, lagarta, pré-pupa e pupa de Dichomeris famulata criadas em estilo-estigmas e grãos de milho. Temperatura de $25 \pm 2^{\circ} \mathrm{C}$, UR de $65 \pm 10 \%$ e fotofase de 14 horas. Piracicaba, SP. Fevereiro de 2007 a fevereiro de 2009.

\begin{tabular}{lccc}
\hline Fase/Período & Duração (dias) & \pm EPM & Viabilidade (\%) \\
\hline Ovo & 4,1 & 0,19 & 63,24 \\
Lagarta & 21,1 & 0,17 & 84,00 \\
Pré-pupa & 1,6 & 0,04 & 100,00 \\
Pupa & 8,4 & 1,21 & 87,97 \\
Ovo-Adulto & 35,2 & & 47,00 \\
\hline
\end{tabular}

duração média de 8,4 dias, com viabilidade de $87,9 \%$ (Tabela 1), diferindo daquela observada por CIRO et al. (1992), cujo valor médio foi de 6 dias. O fato pode ser explicado devido à criação das lagartas ter sido em hospedeiro e temperatura diferentes dos utilizados por aqueles autores. $\mathrm{O}$ peso de pupas, com 24 horas de idade, foi de $12,4 \pm 0,0013 \mathrm{e} 11,3 \pm 0,0011 \mathrm{mg}$, para machos e fêmeas, respectivamente, sendo observada diferença estatística significativa ao nível de $5 \%$ de probabilidade entre sexos. Embora em lepidópteros, as fêmeas, normalmente, pesem mais que os machos (SLANSKY \& SCRIBER, 1985), no presente estudo, observou-se o contrário, estes foram mais pesados. A duração do ciclo biológico (ovo-adulto) de $\boldsymbol{D}$. famulata foi de 35,2 dias, e a viabilidade total de $47,0 \%$ (Tabela 1 ). O resultado é próximo ao encontrado por CIRO et al. (1992), que, em sorgo, obtiveram um valor de 32,8 dias.

Adulto. Os adultos (Figura 1D) possuem $14,9 \pm 0,97 \mathrm{~mm}$ de envergadura, e asas anteriores de coloração marrom levemente esverdeada com manchas escuras. Os palpos labiais apresentam um par de espinhos no ápice e, quando em repouso, as antenas do tipo filiforme se voltam para as asas anteriores. A cópula e a postura são realizadas durante a noite, pois, durante o dia, os adultos permanecem em repouso, porém, quando tocados ou perturbados, ficam agitados. Os períodos de pré-oviposição e de oviposição foram de $10,7 \pm 0,40$ e $14,04 \pm 0,42$ dias, respectivamente. $\mathrm{O}$ período de pré-oviposição foi menor que o relatado por CIRO et al. (1992), ou seja, de 16,18 dias para indivíduos criados em grãos de sorgo. Provavelmente, o período de pré-oviposição das mariposas foi maior porque se alimentaram com uma solução de mel a $0,5 \%$, concentração considerada baixa, pois, segundo PARRA (2007), para lepidópteros, em geral, utiliza-se solução de sacarose ou mel a $10 \%$. As fêmeas colocaram, em

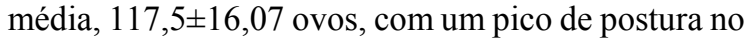
sexto dia de oviposição. A longevidade média de machos e fêmeas foi de $37,02 \pm 5,58$ e 44,16 $\pm 6,50$ dias, respectivamente, diferindo estatisticamente entre os dois sexos. Através dos resultados obtidos, é possível estimar a sobrevivência da espécie em laboratório, pois os dados seguem o modelo de Weibull, sendo que os machos apresentam probabilidade de sobrevivência estimada menor que as fêmeas. Os resultados estão de acordo com os de CIRO et al. (1992), que também observaram uma longevidade maior para as fêmeas em relação aos machos. A razão sexual observada foi de

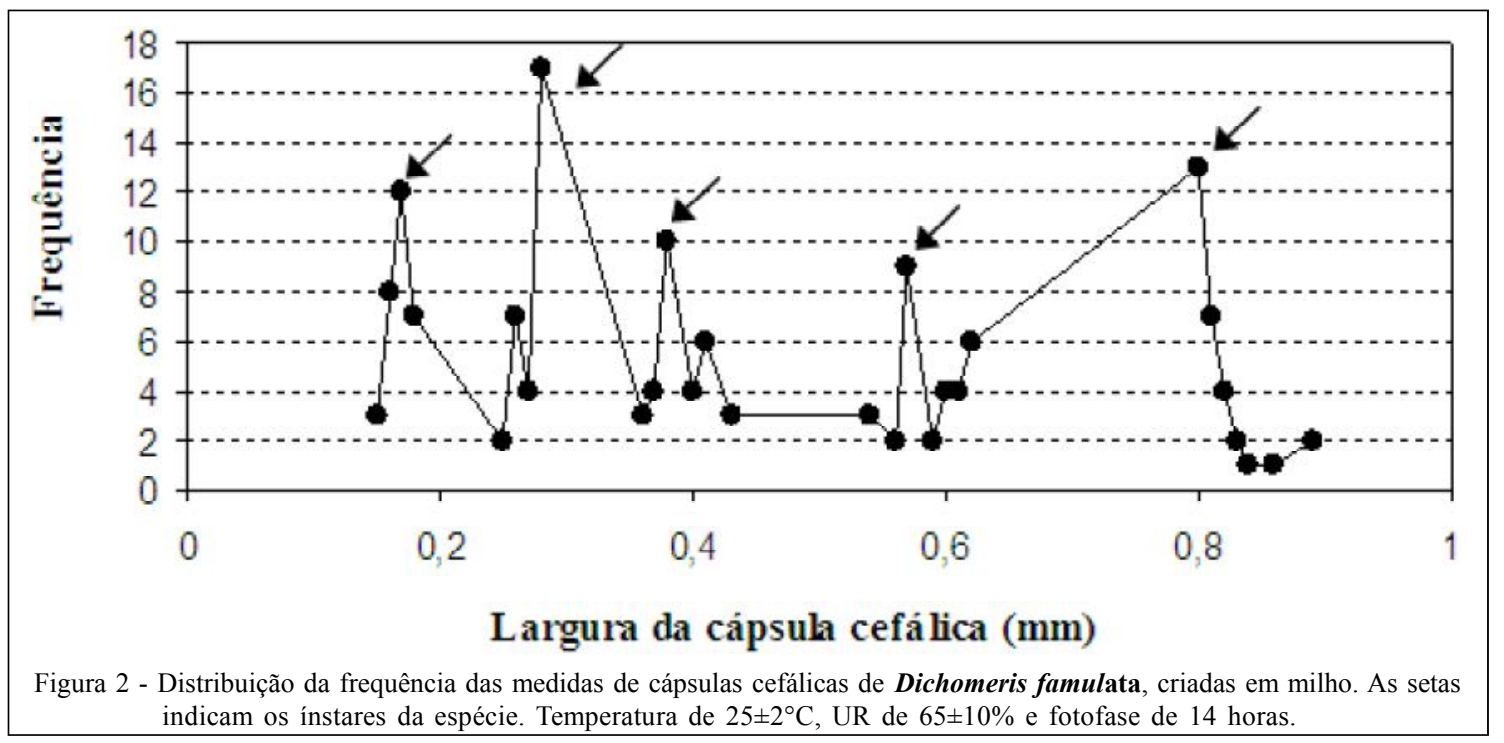

Ciência Rural, v.41, n.1, jan, 2011. 
0,48, uma relação de aproximadamente um macho para cada fêmea.

Ultimamente, a praga vem causando danos nos campos de produção de milho no estado de São Paulo e Minas Gerais por motivos ainda não esclarecidos, mas provavelmente devido ao desequilíbrio ocasionado pelas aplicações inadequadas de agroquímicos para controlar $\boldsymbol{S}$. frugiperda, a lagartado-cartucho do milho (MARQUES, 2009).

O conhecimento da biologia dessa espécie na cultura do milho pode permitir aos pesquisadores e produtores o desenvolvimento e a adoção de métodos de manejo para evitar os seus danos. Estudos futuros sobre a sua dinâmica populacional e comportamento permitirão estabelecer época e melhor forma de controlála, com base nos parâmetros biológicos. Assim sendo, em função do potencial de dano para a lavoura de milhosemente, recomenda-se a inclusão da D. famulata em programas de manejo de pragas da cultura.

\section{CONCLUSÃO}

D. famulata é uma nova praga da espiga do milho. Apresenta ciclo biológico médio de 35 dias, e, na maior parte deste período (21 dias), suas larvas se alimentam dos grãos de milho, provocando injúria que irá afetar a germinação das sementes.

\section{AGRADECIMENTO}

A Coordenação de Aperfeiçoamento de Pessoal de Nível Superior (CAPES), pela concessão da bolsa de mestrado, que permitiu a realização desta pesquisa, ao Prof. Dr. José Roberto Postali Parra, pelas sugestões, ideias e ajuda na biologia do inseto, e ao Dr. Vitor O. Becker, pela identificação da espécie.

\section{REFERÊNCIAS}

BUTT, B.A.; CANTU, E. Sex determination of lepidopterous pupae. Washington: USDA, 1962. 7p. (ARS, 33-75).

CÍCERO, S.M. Mistura de lotes de diferentes idades e sua relação com a qualidade fisiológica e sanidade das sementes e desempenho das plantas de milho (Zea mays L.). 1987. 119f. Tese (Livre-Docência) - Escola Superior de Agricultura "Luiz de Queiroz", Universidade de São Paulo, SP.

CIRO, L.D.R. et al. Ciclo de vida, habitos y enemigos naturales de Dichomeris famulata Meyrick (Lepidoptera: Gelechiidae), plaga de la panoja de sorgo en el Valle del Cauca. Revista Colombiana de Entomologia, Santafe de Bogota, v.18, n.2, p.49-54, 1992. Disponível em: <http://www.scielo.unal.edu.co/ scielo.php?script=sci_serial\&lng=pt\&pid=0120-0488\&nrm=>. Acesso em: 08 out. 2010.

COLOSIMO, E.A.; GIOLO, S.R. Análise de Sobrevivência Aplicada. São Paulo: Edgard Blücher, 2006. 236p.

DYAR, H.G. The number of molts of lepidopterous larvae. Psyche, Cambridge, v.5, p.420-433, 1890. Disponível em:
$<$ http://psyche.entclub.org/5/5-420.html $>$. Acesso em: 08 out. 2010. doi: $10.1155 / 1890 / 23871$.

FNP CONSUTORIA \& COMÉRCIO. Milho. In: Agrianual 2009: anuário da agricultura brasileira. São Paulo, 2009. p.385-406.

HADDAD, M.L. et al. Programa MOBAE: modelos bioestatísticos aplicados à entomologia. Piracicaba: ESALQ/USP, 1995. 44p.

LUIZ, C.B.F.; MAGRO, S.R. Controle biológico das pragas da espiga, sobre parâmetros qualitativos e quantitativos na cultura do milho de safrinha em Ubiratã/PR. Campo Digital, Campo Mourão, v.2, n.1, p.13-21, 2007. Disponível em: <http:// revista.grupointegrado.br/revista/index.php/campodigital/ article/view/320>. Acesso em: 08 out. 2010.

MARQUES, L.H.S.F. Biologia, dano e controle de Dichomeris famulata Meyrick, 1914 (Lepidoptera: Gelechiidae) por meio de iscas. 2009. 78f. Dissertação (Mestrado em Entomologia) - Curso de Pós-graduação em Entomologia, Escola Superior de Agricultura "Luiz de Queiroz", Universidade de São Paulo, SP.

MARTIN, T.N. et al. Questões relevantes na produção de sementes de milho - primeira parte. Revista da Faculdade de Zootecnia, Veterinária e Agronomia - FZVA, Uruguaiana, v.14, n.1, p.119-138, 2007a. Disponível em: <http:// revistaseletronicas.pucrs.br/ojs/index.php/fzva/article/view/ 2483>. Acesso em: 08 out. 2010.

MARTIN, T.N. et al. Questões relevantes na produção de sementes de milho - segunda parte. Revista da Faculdade de Zootecnia, Veterinária e Agronomia - FZVA, Uruguaiana, v.14, n.2, p.80-101, 2007b. Disponível em: <http:// revistaseletronicas.pucrs.br/ojs/index.php/fzva/article/view/ 2501>. Acesso em: 08 out. 2010.

MENEZES, A.P. et al. Ocorrência de Dichomeris famulata Meyrick (Lepidoptera: Gelechiidae) na cultura de milho orgânico solteiro ou consorciado com feijão. Revista Brasileira de Agroecologia, Porto Alegre, v.4, n.2, p.4366-4369, 2009. Disponível em: <http://www.aba-agroecologia.org.br/ojs2/ index.php? journa $1=$ rb a groecologia $\&$ pa ge $=a$ rticle\&op=view \&path[] $=9602 \&$ path []$=6578>$. Acesso em: 08 out. 2010 .

PANIZZI, A.R.; PARRA, J.R.P. Ecologia nutricional de insetos e suas implicações no manejo de pragas. São Paulo: Manole, 1991. 359p.

PARRA, J.R.P. Técnicas de criação de insetos para programas de controle biológico. 6.ed. Piracicaba: FEALQ, 2007. 134p.

SGRILLO, R.B. A distribuição de Weibull como modelo de sobrevivência de insetos. Ecossistema, Espírito Santo do Pinhal, v.7, p.9-13, 1982. Disponível em: <http://189.20.243.4/ ojs/ecossistema/index.php>. Acesso em: 08 out. 2010.

SLANSKY JR., F.; SCRIBER, J.M. Food consumption and utilization. In: KERKUT, G.A.; GILBERT, L.I. Comprehensive insect physiology, biochemistry and pharmacology. Oxford: Pergamon, 1985. p.87-163.

THE R PROJECT FOR STATISTICAL COMPUTING. R version 2.8.1. Acesso em 23 fev. 2009. Online. Disponível em: http://www.r-project.org/. 